\title{
Premodern City Layouts Drawn on Published Maps: A Comparative Analysis of Edo, Osaka, and Kyoto
}

\author{
Akihiro Tsukamoto \\ Tokushima University, tsukamoto.akihiro@tokushima-u.ac.jp
}

Keywords: Historical maps, GIS, layout of City

\begin{abstract}
:
Museums, libraries, and other public research organizations have been creating digital archives of historical maps for some time. Initially, more work was required to archive historical maps than written documents, as they needed to be imaged in sections and then recombined. However, recent improvements in imaging technology and information devices have made it comparatively easy to create digital archives. In recent years, it has even become possible to view and download digital images of historical maps from sites run by the organizations possessing these maps, as well as from cross-searchable portal sites such as Old Maps Online (https://www.oldmapsonline.org) and Map Warper (https://mapwarper.net).
\end{abstract}

These portal sites provide terrestrial georeferencing functionality, in which historical maps are superimposed on a projected coordinate system. This allows the sharing of geometrically corrected historical maps and images, as well as linking table information for control points created during the georeferencing process. As progress is made creating and publishing digital archives of these historical maps, it will become increasingly important to increase both the depth and number of methodologies used to analyze historical maps using GIS.

In this study, I make use of image data from historical maps that have been geometrically corrected as well as control points created during the georeferencing process to conduct a GIS analysis of historical maps of several cities. My specific goal in this study is to analyze historical maps published for Edo, Osaka, and Kyoto, in order to quantitatively reveal differences in the shapes of cities drawn using the unique layouts of historical maps, which differ from modern projected coordinate systems (Table 1).

\begin{tabular}{|c|c|c|c|}
\hline & A & $\mathrm{B}$ & $\mathrm{C}$ \\
\hline Year of publication & 1858 & 1844 & 1863 \\
\hline \multirow{2}{*}{ Publisher } & Suharaya Mohe & Akamatsu Kyube & Kobe Takehara \\
\hline & 須原屋茂兵衞 & 赤松九兵衞 & 竹原好兵衛 \\
\hline \multirow{2}{*}{ Title } & Bunken Edo Oezu: Kan & Zoshu kaisei Sesshu Osaka Chizu: Kan & Shinzo Saiken Kyo Ezu Taizen \\
\hline & 分間江戸大絵図 : 完 & 增脩改正 攝州大阪地圖 : 全 & 新増細見京絵図大全 \\
\hline \multirow{2}{*}{ Current owner organization } & East Asian Library - & East Asian Library - & Art Research Center, \\
\hline & University of California, Berkeley & University of California, Berkeley & Ritsumeikan University \\
\hline Current owner organization code & Ea173 (b104787077) & G22b106232794 & arcBK09-0009 \\
\hline
\end{tabular}

Table 1. Basic information on the analyzed historical maps.

In conducting a comparative analysis for historical maps, map layouts - such as where features are drawn (located) on each map — must be identified from coordinate values. A GIS georeferencing function can be used here to extract errors. The following method was used to measure differences in the coordinate values of features located on historical maps.

1) Two reference points were set near the centers of each historical map that could also be identified on modern maps. Then, using these two points as reference points, georeferencing was performed so that the historical maps for Edo, Osaka, and Kyoto were overlain with modern maps. A similarity transformation was used to correct the historical map images geometrically.

2) Coordinate values were obtained for the landmark points on each historical map. The landmark points (control points) used for comparison during this study could be identified on both the modern and historical maps First, 116 points were set for Edo, 229 points for Osaka, and 163 points for Kyoto. Next, the difference in the coordinate values for the comparable points on each modern map was calculated (Table 2).

3) The distance between two features that are the same on both the historical map and modern map were visualized on each historical map. The inverse distance weighted (IDW) method was used here to perform space interpolation in order to summarize errors in a more easily understood manner (Figure 1). 
Finally, based on the procedure described above, an investigation of the layout of features located on maps based on the differences in positional coordinates between the historical and modern maps was performed. Table 2 summarizes information obtained after calculating the distance between the same features on modern maps and each drawing. Note that these distances are the same as the residual errors for each control point set during georeferencing. Table 2 shows the error tendency for each historical map. Osaka (historical map B) was drawn with the most accuracy, while the drawing of Kyoto (historical map C) contains significant distortion.

\begin{tabular}{lrrr} 
& A & B & \multicolumn{1}{l}{ C } \\
\hline \hline Average & 554.46 & 86.52 & 2430.63 \\
\hline Standard deviation & 258.15 & 76.81 & 2884.76 \\
\hline Standard error & 23.97 & 5.08 & 225.95 \\
\hline Number of samples & 116 & 229 & 163 \\
\hline
\end{tabular}

Table 2. Error tendency in the analyzed historical maps.

Errors on historical maps were then visualized in more detail to determine where significant errors occurred and which areas were drawn accurately (Figure 1). In Edo (historical map A), errors tended to increase moving from the area around Edo Castle (which was set as a reference point) to the outskirts of the area. In Osaka (historical map B), errors were minor throughout the map, with the urbanized area and westward sections being particularly accurate. This suggests that the artists who drew historical maps A and B worked hard to reproduce the actual areas as realistically and in as much detail as possible when drawing their maps. In Kyoto (historical map C), however, the city area (inside the capital) in the center accounted for more than half of the paper map, with many temples, shrines, and historic places located around the city area packed into the empty spaces on the edge of the paper. Therefore, the outskirts of the map show a distribution of landmarks far removed from their actual location, resulting in significant errors. It is clear that this map is meant to show the unique layout of Kyoto and does not reflect the results of any kind of survey, as in the case of the maps for Edo and Osaka.
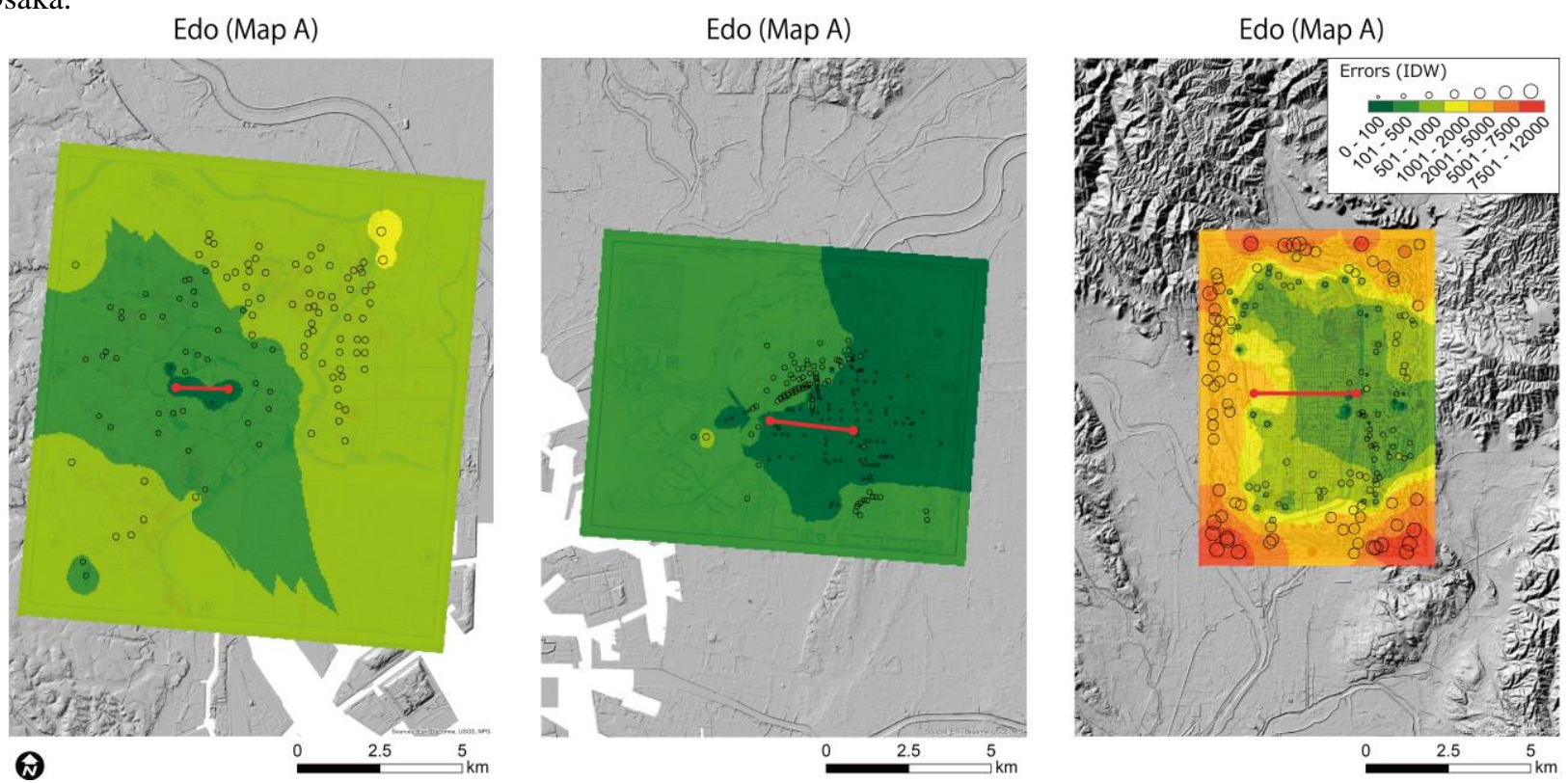

Figure 1. Errors between modern maps and historical maps

(Errors between modern maps and historical maps were interpolated via IDW and visualized on the historical maps)

Maps drawn based on survey results began circulating throughout society during the mid-19th century. Although the maps for Osaka and Edo were drawn taking these survey results into consideration, the map for Kyoto was drawn with the same layout used since the 17th century. It is likely the case that artists chose whether to change or keep city layouts on maps, rather than always striving to create more accurate maps as time went on.

During this study, I made use of GIS spatial analysis functionality to analyze historical maps created around 1840 in order to analyze the extent to which artists were able to represent actual spaces on pieces of paper of limited size and was able to clarify partial city layouts. Further analysis will need to be conducted by taking insight from prior research on published maps in the fields of historical geography and historical map research into consideration.

Acknowledgments: This work was supported by 2017-2020 Grants-in-Aid for Scientific Research: Young Researchers (B) "Historical GIS Research on Premodern/Modern Published Map Layouts and Lineages" (representative researcher: Akihiro Tsukamoto) and 2016-2019 Grants-in-Aid for Scientific Research: Foundational Research (A) "Development of Digital Humanities Using Historical GIS" (representative: Keiji Yano) 